\title{
Temporary CXCR3 and CCR5 Antagonism Following Vaccination Enhances Memory CD8 T Cell Immune Responses
}

\author{
Rui Li, ${ }^{1}$ Nan Zhang, ${ }^{1^{*}}$ Miaomiao Tian, ${ }^{1^{*}}$ Zihan Ran, ${ }^{1}$ Mingjun Zhu, ${ }^{1}$ Haiyan Zhu, ${ }^{2}$ Fangting Han, ${ }^{1}$ \\ Juan Yin, ${ }^{1}$ and Jiang Zhong $^{1}$ \\ ${ }^{1}$ Department of Microbiology and Microbial Engineering, School of Life Sciences; and ${ }^{2}$ Department of Biosynthesis, School \\ of Pharmacy, Fudan University, Shanghai, China
}

\begin{abstract}
Although current vaccination strategies have been successful at preventing a variety of human diseases, attempts at vaccinating against some pathogens such as AIDS and tuberculosis (TB) have been more problematic, largely because abnormally high numbers of antigen-specific CD8 + T cells are required for protection. This study assessed the effect on host immune response of temporarily dampening the chemokine receptors CXCR3 and CCR5 after vaccination by administration of TAK-779, a small-molecule CXCR3 and CCR5 antagonist commonly used to inhibit HIV infection. Our results showed that use of TAK-779 enhanced memory CD8 + T cell immune responses both qualitatively and quantitatively. Treatment with TAK-779 following vaccination of an influenza virus antigen resulted in enhanced memory generation, with more CD8 + CD $127+$ memory precursors and fewer terminally differentiated effector CD8 + CD69 + T cells. These memory T cells were able to become IFN- $\gamma$-secreting effector cells when re-encountering the same antigen, which can further enhance the efficacy of vaccination. The mice vaccinated in the presence of TAK-779 were better protected upon influenza virus challenge than the controls. These results show that vaccination, while temporarily inhibiting chemokine receptors CXCR3 and CCR5 by TAK-779, could be a promising strategy to generate large numbers of protective memory CD8 + T cells.

Online address: http://www.molmed.org
\end{abstract}

doi: $10.2119 / \mathrm{molmed} .2015 .00218$

The ability to develop and sustain populations of memory $T$ cells after infection or immunization is a hallmark of the adaptive immune response and a basis for protective vaccination against infectious disease. Although current vaccination strategies have been successful at preventing a variety of human diseases, a number of major hurdles remain. In particular, attempts at vaccinating against malaria, AIDS

\section{INTRODUCTION}

and tuberculosis (TB) have been more problematic, largely because abnormally high numbers of antigen-specific CD8 + T cells are required for protection. (1)

During an acute peripheral infection, $\mathrm{T}$ cells first become activated in the lymph nodes (LNs) and spleen, and then gain the ability to migrate to the site of infection through a complex series of interactions involving adhesion molecules and chemokine receptors. Upon antigen

*Both authors contributed equally to the work

Address correspondence to Rui Li, Department of Microbiology and Microbial Engineering, School of Life Sciences, Fudan University, 2005 Songhu Road, Shanghai 200438, P.R. China. Phone: +86 21 51630660; Fax: +86 21 51630661; E-mail: rui_li@fudan.edu.cn; or Jiang Zhong, Department of Microbiology and Microbial Engineering, School of Life Sciences, Fudan University, 2005 Songhu Road, Shanghai 200438, China. Phone: +86 21 5163066 1; Fax: +86 21 51630667; E-mail: jzhong@fudan.edu.en.

Submitted October 2, 2015; Accepted for Publication June 29, 2016; Published Online (wWw.molmed.org) July 6, 2016.

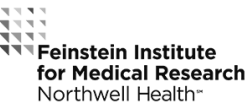

encounter, antigen-specific CD8 + T cells undergo rapid clonal expansion and differentiate into cytotoxic effector $\mathrm{T}$ cells, which later play an essential role in infection control through lysis of the infected cells and production of cytokines. (2) After the peak of expansion and pathogen clearance, the effector CD8 + T cell pool undergoes extensive contraction, which eliminates 90-95\% of pathogen-specific effector CD8 $+\mathrm{T}$ cells. $(2,3)$ The remaining $5-10 \%$ of pathogen-specific CD8 + cells survive to become long-lived memory cells. $(2,3)$

The magnitude and quality of the memory CD8 $+\mathrm{T}$ cell population are shaped and influenced by the strength and duration of the initial antigenic stimulus as well as by inflammatory cytokines. Although there is compelling evidence that the inflammatory signals are crucial for clonal expansion, effector CD8 + T cell differentiation and memory development, excessive and prolonged exposure to inflammatory signals is detrimental to 
generating potent memory CD8 + T cells. $(4,5)$ Actually, a preponderance of data supports a crucial role for the strength of inflammatory stimuli during the early expansion phase in controlling effector vs memory cell-fate decisions of CD8 + T cells. For example, studies have shown that too much inflammation, as from high levels of proinflammatory cytokines such as IL-12 and IFN- $\gamma$, favors the generation of terminally differentiated short-lived effector CD8 + T cells (SLECs). (6-8) On the other hand, homeostatic cytokines such as IL-7 and IL-15 promote the formation of memory precursor effector CD8 + T cells (MPECs). $(9,10)$ In particular, recent studies have shown that CXCR3 chemokine receptors are involved in promoting CD8 + T cell commitment to an effector fate rather than a memory fate. $(11,12)$ In addition, Kohlmeier et al. found that $\mathrm{Ccr}^{-/-} \mathrm{Cxcr}^{-/-}$ cells exhibited markedly decreased contraction and an accumulation of massive numbers of memory CD8 + T cells after infection, where CXCR3 is primarily responsible for this phenotype. (13) These studies underscore the role of inflammatory chemokine receptors in the generation of CD8 + T cell memory.

In the present study, we investigated for the first time the impact of temporary CXCR3 and CCR5 inhibition following vaccination on host immune responses, especially CD8 + T cell immune responses, in murine models. TAK-779, a nonpeptide, synthetic, small-molecule CCR5 and CXCR3 antagonist, was used along with an influenza virus nucleoprotein (NP). TAK-779 was initially developed for the treatment of HIV infection, whereby CCR5 functions as a major coreceptor for fusion and entry of macrophage-tropic HIV-1 into the host cells. (14) Furthermore, TAK-779 has also been shown to have the ability to block binding of CXCR3 and inhibitory protein 10, one of the CXCR3 ligands. (15) Targeting CCR5 and CXCR3, TAK-779 has been used to inhibit allograft rejection (16-18), inflammatory bowel disease (19), experimental autoimmune encephalomyelitis (EAE) and airway hyperresponsiveness and inflammation. (20) Our result is of great significance to vaccinations against certain pathogens, such as HIV, Mycobacterium tuberculosis bacteria and malaria parasite, where abnormally high numbers of antigen-specific CD8 + T cells are required for protection. In addition, our study provides supporting information on the immunomodulatory and beneficial use of Maraviroc (MVC)-containing antiretroviral therapy (cART) in HIV-infected patients.

\section{MATERIALS AND METHODS}

\section{Animals}

We obtained 4- to 5-wk-old female BALB/c mice from the Animal Center of Slaccas (Shanghai, China). The mice were kept under specific pathogen-free (SPF) conditions in individual ventilated cages (IVCs). All animal care and experimental procedures were carried out according to the National Institutes of Health Guide for Care and Use of Laboratory Animals, and were approved by the Bioethics Committee of Fudan University.

\section{Construction of NP Expression Vector and Expression and Purification of NP Protein}

A full length of influenza virus NP segment was amplified from plasmid containing $n p$ gene (A/ chicken/Nakorn-Patom/Thailand/ CU-K2/2004(H5N1)), provided by Prof. Alonso Sylvie (National University of Singapore), using primers 5'-TTCCATGGATGGCGTCTCAAGGCACCAAAC-3' and 5'-TTGAATTCTTAATTGTCGTACTCCTCTGCATTG-3' (NcoI and EcoRI sites are underlined). The resulting PCR fragment was then cloned into pMD-18T vector, yielding pMD18T-PR8-NP-, and was further confirmed by PCR screening and sequencing. NP fragment was subsequently cloned into prokaryotic expression vector pET-30a(+) (Novagen) double-digested with NcoI and EcoRI (TaKaRa), yielding pET-30a-NP. Positive clones were confirmed by PCR and sequencing.

NP expressing plasmid pET-30a-NP was transformed into Escherichia coli strain BL21 (DE3) and a single colony was inoculated into the LB liquid medium containing kanamycin. After IPTG induction at $25^{\circ} \mathrm{C}$, bacteria were collected by centrifugation and resuspended in binding buffer for ultrasonication. The supernatant containing NP protein was then purified by Ni Sepharose affinity chromatography. Purified NP protein was dissolved in phosphate buffered saline (PBS) and then subjected to SDS-PAGE. Western blotting was later performed using 1:5,000 diluted mouse anti-His tag monoclonal antibodies (Sigma) and 1:30,000 diluted goat-anti-mouse Abs-conjugated to alkaline phosphatase (Sigma).

\section{Mouse Treatments}

TAK-779, kindly provided by Dr. Zhisong Chen from the National Institutes of Health, was dissolved in 5\% mannitol solution to a concentration of $1.5 \mathrm{mg} / \mathrm{mL}$. After filtration sterilization, TAK-779 solution was aliquoted in sterile EP tubes and stored at $-20^{\circ} \mathrm{C}$. Mouse immunization and treatment schedules are illustrated in Figure 1. The mice received intraperitoneal immunization once or twice at a 4 -wk interval with $40 \mu \mathrm{g}$ NP protein emulsified with Freund's incomplete adjuvant (IFA). Each mouse in TAK-779-treated groups was injected subcutaneously on the left, right or middle abdomen with $150 \mu \mathrm{g}$ TAK-779 in a volume of $100 \mu \mathrm{L}$, once daily after NP immunization. Control mice were injected subcutaneously with PBS instead of TAK779. TAK-779 administration was started from day 0 after immunization and continued for $4 \mathrm{wks}$ or $8 \mathrm{wks}$ for 1 or $2 \mathrm{immu-}$ nizations, respectively. The dose of $150 \mu \mathrm{g}$ per mouse was chosen based on previous publications showing that this dose was successfully used in experimental EAE (21) and collagen-induced arthritis (22), and approximately the same dose was used in other disease experiments, such as allograft rejection and asthma models. $(20,23)$ For influenza virus infection, 2 wks after stopping TAK-779, sedated mice received intranasal (i.n.) administration of $1050 \%$ lethal doses (LD50) of H1N1 (A/FM/1/47) in sterile RPMI 1640 cell culture media supplemented with penicillin 
A

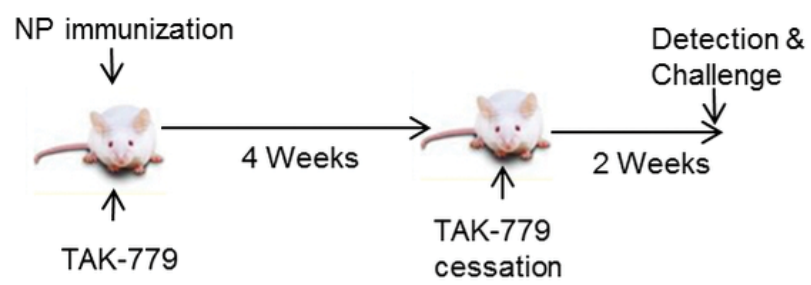

B

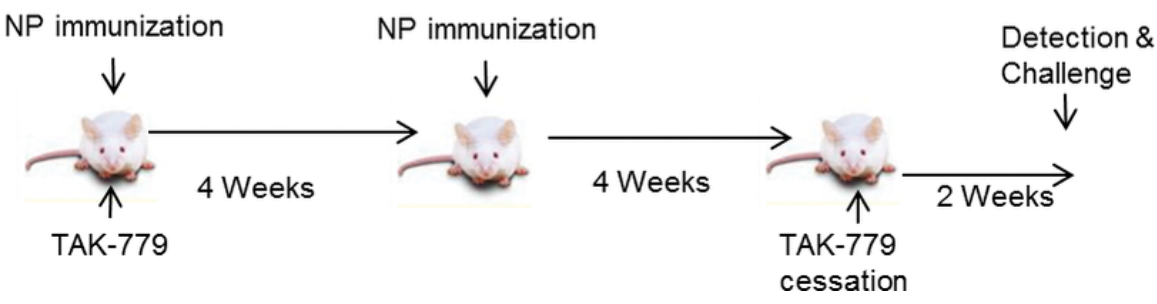

Figure 1. Mouse immunization and treatment schedules. The mice were immunized intraperitoneally once or twice at a 4 wk interval with $40 \mu \mathrm{g}$ NP protein emulsified with Freund's incomplete adjuvant (IFA). TAK-779 administration was started from d 0 after immunization and continued once daily for 4 wks or 8 wks for 1 or 2 immunizations, respectively. For influenza virus infection, 2 wks after stopping TAK-779, sedated mice received intranasal (i.n.) administration with $1050 \%$ lethal doses (LD50) of H1N1 (A/FM/1/47).

and streptomycin. Ten mice per group were used to determine the survival rates based on body weight loss, and the mice were euthanized when body weight loss exceeded $25 \%$ of original body weight.

\section{Antibody Detection}

Serum was collected by retro-orbital bleeds from immunized mice, and stored at $80^{\circ} \mathrm{C}$ for subsequent experiment. The presence of antibodies in the serum was measured by ELISA. Ninety-six-well microtiter plates (Costar; Corning) were coated overnight at $4^{\circ} \mathrm{C}$ with $100 \mu \mathrm{L}$ of $0.1 \mathrm{M}$ carbonate buffer ( $\mathrm{pH}$ 9.6) containing $1 \mu \mathrm{g} / \mathrm{mL}$ of NP. After blocking with $2 \%$ bovine serum albumin (BSA) in PBS containing $0.1 \%$ Tween $20,100 \mu \mathrm{L}$ of serial diluted serum $\left(1: 1 \times 10^{4}-1: 1.28 \times 10^{6}\right)$ was added to the wells. The plates were incubated at $37^{\circ} \mathrm{C}$ for $1.5 \mathrm{~h}$, rinsed in PBS- $0.1 \%$ Tween 20, and incubated at $37^{\circ} \mathrm{C}$ for $1 \mathrm{~h}$ with $50 \mu \mathrm{L}$ of horseradish peroxidase (HRP)-conjugated goat anti-mouse IgG $(\mathrm{H}+\mathrm{L})$ (Sigma) diluted $1: 10,000$. To detect the various IgG subtypes, HRP-conjugated goat anti-mouse IgG1, IgG2a and IgG3 secondary antibodies (Abcam) were diluted at 1:30,000.
The reaction was then developed with $100 \mu \mathrm{L}$ of TMB liquid substrate solution (T4444, Sigma) at room temperature for $30 \mathrm{~min}$ in the dark and stopped by the addition of $1 \mathrm{M}$ sulfuric acid. The absorbance at $450 \mathrm{~nm}$ was measured by an ELISA plate reader (Tecan Sunrise).

\section{Flow Cytometry Analysis}

Spleens from individual mice were harvested and single cell suspensions were prepared by meshing the spleens through $70 \mu \mathrm{M}$ cell suspension mesh (BD), followed by centrifugation on Ficoll-PaqueTMPLUS (GE) for $20 \mathrm{~min}$ at $600 \times \mathrm{g}$ at room temperature. Cells were collected and washed once with sterile FACS buffer (2\% FCS, 5 mM EDTA in PBS). Splenocytes $\left(10^{6}\right)$ were stained with the following antibodies: Anti-Mouse CD3e-PE-cy ${ }^{\mathrm{TM}}$ 7, Anti-Mouse CD4-FITC, Anti-Mouse CD8a-APC, Anti-Mouse CD127-FITC, Anti-Mouse CD69-PE or isotype control. All antibodies were bought from BD Pharmingen. Data were collected on a BD FACS Aria flow cytometer (BD Bioscience) and analyzed with Flowjo Software (Treestar Inc.). Gating strategies for FACS analysis are shown in Figure S1.

\section{Reverse Transcription-polymerase Chain Reaction (PCR) and Quantitative Real-time RT-PCR}

Individual spleen pieces were collected 2 wks after TAK-779 treatment and stored at $-80^{\circ} \mathrm{C}$ for further analysis. Total RNA was isolated from whole spleen using Trizol reagent (Gibco-BRL) according to the manufacturer's protocol. Total RNA (500 ng) was reverse transcribed using PrimeScript ${ }^{\mathrm{TM}}$ RT Master Mix (Takara Bio, Japan) following the manufacturer's instructions. Aliquots $(2 \mu \mathrm{L})$ of the RT product were quantified by SYBR Green 2-step real-time RTPCR on QuantStudio ${ }^{\text {TM }} 7$ flex Real-Time PCR system (ABI, USA), according to the manufacturer's instructions. The mRNA levels were normalized to those of $\beta$-actin. The PCR primer sets used in this study were: CCL4 sense, 5'-TTCTGTGCTCCAGGGTTCTC-3'; CCL4 antisense, 5'-CGGGAGGTGTAAGAGAAACAG-3'; CCL5 sense, 5'-ATCTTGCAGTCGTGTTTGTCA-3'; CCL5 antisense, 5'-TTCTTGAACCCACTTCTTCTCTG-3'; CXCL9 sense, 5'-GCCTTCTTGGCGTAGGTATTG-3'; CXCL9 antisense, 5'-ATCGGTTGAGGAAAGTGATGC-3'; CXCL10 sense, 5'-CGATAAACGTCCCTCCCGTAA-3'; CXCL10 antisense, 5' - CAGACCCGTCCCTATCTTTCC-3'; CCR5 sense, 5'-CGGAACTTCTCCCCAACAAA-3'; CCR5 antisense, 5'-CTTTCTCTTCTGGACTCCCTACAACA-3'; CXCR3 sense, 5'-CCAGAATAAATGACAGGGCACAA-3'; CXCR3 anti-sense, 5'-AAGAAAGGCAAAGTCCGAGGC-3'; $\beta$-actin sense, $5^{\prime}$ - TCCTGAGCGCAAGTACTCTGT-3'; $\beta$-actin antisense, $5^{\prime}$ CTGATCCACATCTGCTGGAAG-3'.

\section{Measurement of Proliferation by Carboxyfluorescein Succinimidyl Ester Dilution \\ Carboxyfluorescein succinim-} idyl ester (CFSE) is a cell-permeant fluorescein-based dye that covalently attaches to cytoplasmic components of cells, resulting in uniformly bright fluorescence. CFSE has the ability to stably label molecules within cells, with each cell division resulting in a sequential 
halving of fluorescence. Usually, lymphocyte proliferation can be monitored by flow cytometry for up to 8 divisions before CFSE fluorescence is decreased to the background fluorescence level of unlabeled cells. (24) CFSE labeling was performed as previously described. (24) Briefly, splenocytes were adjusted to $2 \times 10^{6}$ cells $/ \mathrm{mL}$ followed by 3 washings in PBS and incubated with CFSE (ebioscience) in PBS (labeling concentration of $2.5 \mu \mathrm{M}$ ) for $5 \mathrm{~min}$ at room temperature in the dark. The labeling reaction was stopped by adding 10 volumes of PBS containing $2 \%$ heat-inactivated fetal calf serum (HI FCS) $\left(20^{\circ} \mathrm{C}\right)$, and sedimented by centrifugation at $300 \times \mathrm{g}$ for $5 \mathrm{~min}$ at $20^{\circ} \mathrm{C}$. Cells were washed twice more with PBS to remove the excess CFSE and resuspended in complete RPMI 1640 medium. Labeled cells were seeded into 24 well plates at a concentration of $10^{6} / 500 \mu \mathrm{L} /$ well and cultured in the presence of $40 \mu \mathrm{g} / \mathrm{mL} \mathrm{NP}$ protein as a stimulating antigen or medium alone for $3 \mathrm{~d}$. Upon harvest, cells were washed once with PBS and stained with Anti-Mouse CD8a-APC and isotype controls at $4^{\circ} \mathrm{C}$ for 10 mins. Flow cytometry was performed using BD FACS Aria (BD Bioscience) and analyzed with Flowjo software. Cell proliferation was measured as mean fluorescence intensity (mfi) of CFSE. Gating strategies for FACS analysis are shown in Figure S2.

\section{IFN- $\gamma$ ELISpot Assay}

The frequency of antigen-specific IFN- $\gamma$-producing cells was determined by ELISpot assay using a mouse ELISpot set (BD Pharmingen) according to the manufacturer's instructions. Briefly, single-cell suspensions of individual spleens from naïve, TAK-779-treated, NP-immunized and NP-immunized plus TAK-779treated mice were plated in 96-well microplates (Millipore) precoated with $100 \mu \mathrm{L}$ of $5 \mu \mathrm{g} / \mathrm{mL}$ anti-IFN- $\gamma$ antibody in sterile PBS overnight at $4^{\circ} \mathrm{C}$, washed 3 times and blocked for $2 \mathrm{~h}$ at room temperature with RPMI 1640 containing $10 \%$ FCS. Cells were then incubated with $50 \mu \mathrm{g} / \mathrm{mL}$ of NP protein or with
$5 \mu \mathrm{g} / \mathrm{mL}$ conA for $36 \mathrm{~h}$ at $37^{\circ} \mathrm{C}$ in a $5 \%$ $\mathrm{CO}_{2}$ atmosphere. The plates were then washed, followed by addition of biotinconjugated anti-mouse IFN- $\gamma$ antibody for $2 \mathrm{~h}$ at room temperature. After washing, streptavidin-HRP conjugate was added and incubated at room temperature for $1 \mathrm{~h}$. Wells were washed again and developed with a 3-amino-9-ethyl-carbazole (AEC) substrate solution until spots were visible. After drying, spot-forming cell numbers were counted by Bioreader ${ }^{\circledR}$ 4000 (Biosystem). Six animals per group were individually assayed.

\section{Determination of the Viral Titers}

Mouse lungs were harvested and homogenized using mechanical disruption (Omni homogenizer), and tested for the presence of viable virus by tissue culture infectious dose 50 (TCID50) assay using the method described previously. (25) Briefly, 90\% confluent Madin-Darby canine kidney (MDCK) cells in 96-well plates were inoculated with $100 \mu \mathrm{L}$ of 10-fold serially diluted lung homogenates. Plates were incubated at $35^{\circ} \mathrm{C}$ in a humidified incubator $\left(5 \% \mathrm{CO}_{2}\right)$ for $3 \mathrm{~d}$. TCID50 was determined by a reduction in cytopathic effect (CPE) of 50\%, and the $\log$ TCID50/lung was derived. Five mice per group per time point were individually assessed.

\section{Histopathological Examination}

Different groups of mice were immunized and treated as described and challenged with 10 LD50 H1N1 (A/FM/1/47), as indicated in Figure 1. The mouse lungs were harvested $3 \mathrm{~d}$ after challenge and fixed in 10\% formalin in PBS and embedded in paraffin, sectioned and stained with hematoxylin and eosin (H\&E). Observations were made using an inverted light microscope at $\times$ 10 and $\times 40$ objectives.

\section{Statistical Analysis}

Unless otherwise stated, bars represent means $\pm \mathrm{SD}$, and averages were compared using a bidirectional unpaired Student $\mathrm{t}$ test with a $5 \%$ significance level with ${ }^{*} p \leq 0.05,{ }^{* *} p \leq 0.01$ and ${ }^{* * *} p \leq 0.001$.

\section{RESULTS}

\section{Expression and Purification of NP in $E$. coli}

Full-length influenza A virus NP protein was expressed in E. coli BL21 after $0.02 \mathrm{mM}$ IPTG induction at $25^{\circ} \mathrm{C}$. Most of the proteins were found in soluble form when NP was expressed at $25^{\circ} \mathrm{C}$. Purification was performed by Ni Sepharose affinity chromatography under nondenaturing condition to a purity of more than 98\% (Figure 2A). SDS-PAGE analysis showed a monomeric band at approximately 56 kDa (Figure 2A), which was further confirmed by western blotting using mouse anti-His tag monoclonal antibodies (Figure 2B).

\section{TAK-779 Treatment Did Not Change Humoral Immune Response to NP}

Mice were immunized and treated with TAK-779, as illustrated in Figure 1. Following immunization, both the NP immunization only group and the NP immunization plus TAK-779 treatment (TN) group had significantly increased anti-NP IgG antibody titers at the time of detection (Figures 3A, B). However, there was no significant difference in anti-NP IgG antibody titers between the NP only and NP plus TAK-779 groups. Further IgG isotyping also showed that both groups had significant levels of IgG1 antibodies (Figures 3C, D). This was the same for mice groups with $2 \mathrm{NP}$ immunizations (Figure S3). Taken together, our results suggest that TAK-779 did not affect humoral immune responses to immunized antigens (Figure 3).

\section{Treatment with Tak-779 Following Vaccination did not Affect CD4 + T Cell Response,but Resulted in Enhanced Memory Generation, with More Memory Precursor and Fewer Terminally Differentiated Effector CD8 + T Cells}

Previous studies have shown that the chemokine receptors CXCR3 and CCR5 play important roles in regulating effector CD8 + T cell contraction and memory generation after infection. (11-13) We next examined the phenotypes of CD8 $+\mathrm{T}$ 
A

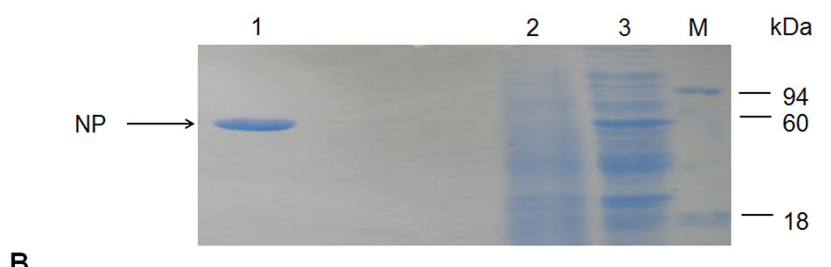

B

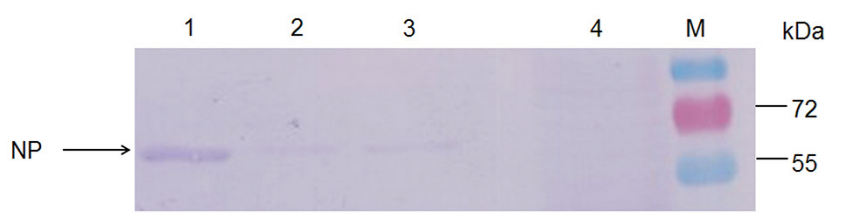

Figure 2. SDS-PAGE and western blot analysis of purified full-length NP protein expressed in Escherichia coli. (A) SDS-PAGE and Coomassie staining. Lane 1: purified NP protein; lane 2: bacterial whole cell lysate without IPTG induction; lane 3: bacterial whole-cell lysate with IPTG induction. (B) Western blot analysis. Purified NP protein and uninduced bacterial whole-cell lysate were detected with 1:5,000 diluted mouse anti-His tag monoclonal antibodies (Sigma) and 1:30,000 diluted goat-anti mouse Abs-conjugated to alkaline phosphatase (Sigma). Lanes 1, 2, 3: purified NP protein; lane 4: bacterial whole-cell extract without IPTG induction.
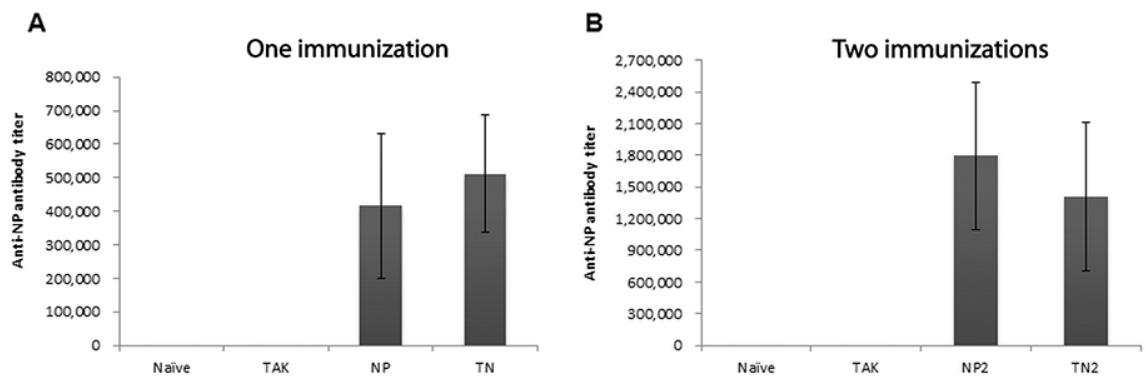

C
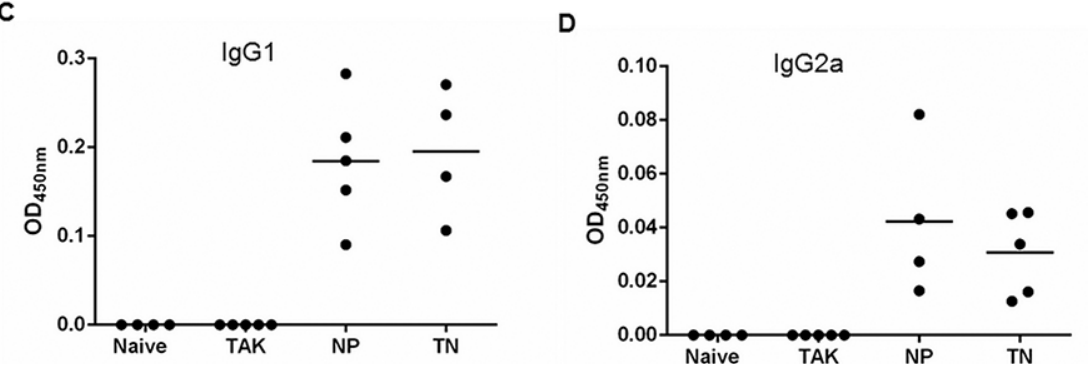

Figure 3. Anti-NP immune responses in mice immunized with NP with or without TAK-779 treatment. Two weeks after TAK-779 cessation, total anti-NP IgG antibody titers were detected by ELISA in sera from naïve mice, mice treated with TAK-779 (TAK), mice immunized once (NP) or twice with NP (NP2) and mice immunized once (TN) or twice (TN2) with NP and treated with TAK-779. (A) One NP immunization; (B) two NP immunizations. $\lg G 1$ (C) and lgG2a (D) isotyping were carried out for mice from naïve, TAK, NP and TN groups. Sera were diluted 1:10,000. cells in mice after NP immunization with or without TAK-779 administration. Two weeks after TAK-779 treatment, splenocytes were prepared from different mice groups and analyzed by flow cytometry after staining with different markers. As shown in Figure 4A, CD8 + T cells in NP immunized plus TAK-779 treated mice (TN group) had characteristics of high memory potential compared with their non-TAK-779 treated counterparts, as determined by expression of memory marker CD127. $(26,27)$ This difference became greater for mice immunized twice with NP along with TAK-779 treatment (TN2 group) (Figure 4C). In contrast, significantly fewer CD8 + T cells in the TN group mice expressed the activation marker CD69 (Figures 4B, D), suggesting that compared with the CD8 + cells of the NP group, these cells were exposed to relatively weak inflammatory/antigenic stimuli in vivo, resulting in shortened expression of the activation marker CD69 during early expansion, followed by enhanced differentiation into MPECs.

Although previous studies have provided concrete evidence that TAK-779 specifically targets CCR5 and CXCR3, to verify that the observed enhanced memory generation was indeed due to TAK-779 treatment, we further analyzed the expression of mRNA for CCR5 and CXCR3, as well as their ligands CCL4 (MIP-1 $\beta$ ), CCL5 (RANTES), CXCL9 (Mig) and CXCL10 (IP-10) in the spleens of mice immunized twice with NP with or without TAK-779 treatment. Spleen samples were collected 2 wks after TAK-779 treatment. Expression of CCR5 and CXCR3, as well as CCL4, CCL5, CXCL9 and CXCL10, was markedly decreased in the spleens of NP-immunized TAK-779-treated mice compared with NP-immunized non-TAK779-treated mice (Figure 5).

Considering the potential influence of CD4 + T cells on CD8 + T cells, we also evaluated CD4 $+\mathrm{T}$ cell response after TAK-779 treatment in mice immunized once or twice with NP. As shown in Figures 4E and F, CD4 + T cell response was unaffected by TAK-779 treatment in mice immunized once or twice with NP. 

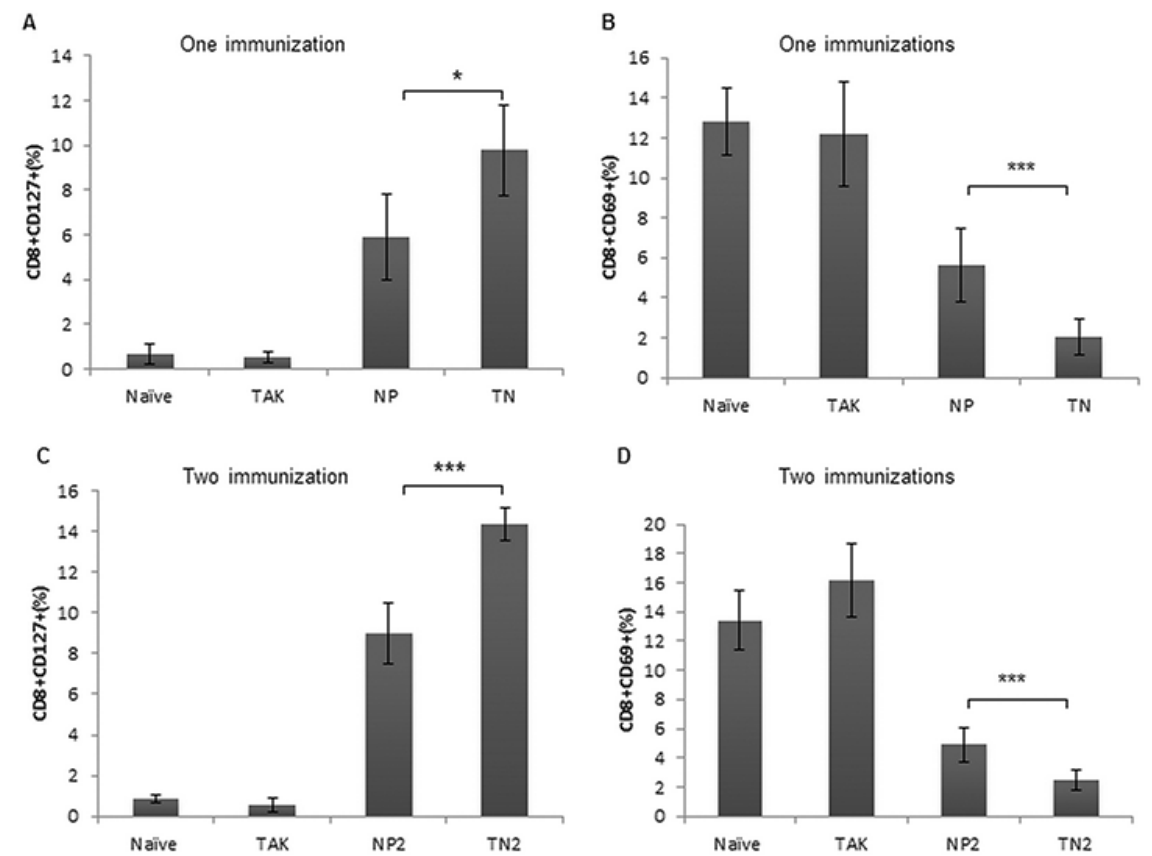

D

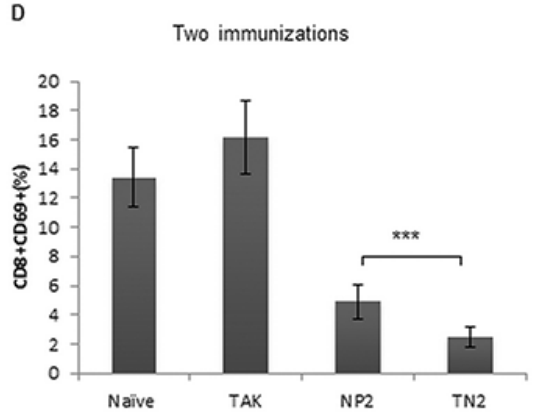

$\mathrm{E}$

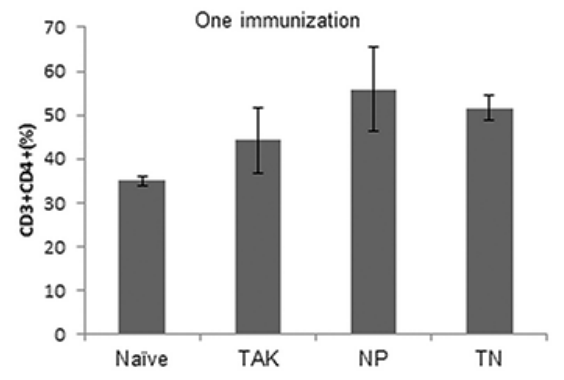

$\mathrm{F}$

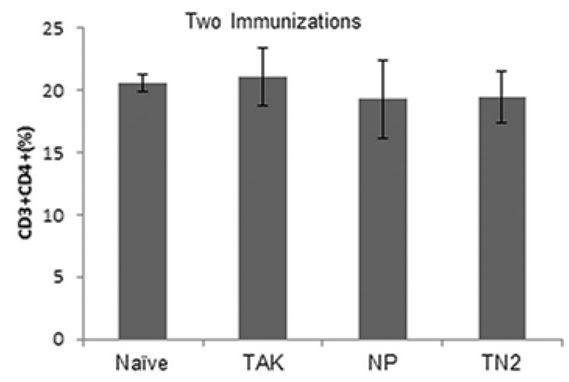

Figure 4. Staining of CD127 and CD69 on CD8 cells and detection of CD4 + cells from NP immunized mice with or without TAK-779 treatment. Two weeks after TAK-779 cessation, splenocytes were prepared from different mice groups and analyzed by flow cytometry after staining with anti-Mouse CD8a-APC, anti-Mouse CD127-FITC, anti-Mouse CD69-PE, anti-Mouse CD3e-PE-Cy ${ }^{\mathrm{TM}} 7$, anti-Mouse CD4-FITC or isotype control antibodies. Percentage of CD8 + CD127 + (A and $C)$ and CD8 + CD69 + (B and D) cells in mice immunized once ( $A$ and $B$ ) or twice ( $C$ and $D)$ with NP with or without TAK-779 treatment were analyzed by flow cytometry and compared with mice from the control group. Percentage of CD3 + CD4 + cells immunized once or twice with NP is illustrated in (E) and (F), respectively. NP: 1 NP immunization; NP2: 2 NP immunizations; TN: 1 NP immunization with TAK-779 treatment; TN2: 2 NP immunizations with TAK-779 treatment. Results are expressed as the mean $\pm \mathrm{SD}$. ${ }^{*} p \leq 0.05 ;{ }^{* * *} p \leq 0.001$. The data are representative of 2 independent experiments with at least 4 mice per group. Mice were analyzed individually.

\section{TAK-779 Treatment Did Not Enhance the Proliferative Ability of CD8 + T Cells in NP-Immunized Mice Treated with TAK-779}

To investigate whether the increase in CD8 + memory cells in NP-immunized mice after TAK-779 treatment was due to higher homeostatic proliferation, we analyzed the proliferative ability of CD $8+\mathrm{T}$ cells after CFSE labeling. The division profiles of CD8 + T cells from NP-immunized TAK-779-treated mice are similar to those of their non-TAK-779-treated counterparts (Figure 6). These data demonstrate that although TAK-779 treatment resulted in quantitative and phenotypic differences in CD8 + T cells, it did not enhance the proliferative ability of CD8 + T cells.

\section{CD8 + T Cells Generated after TAK- 779 Treatment in NP-Immunized Mice Showed Effector Functions in Response to Antigenic Stimulation}

Given the marked increase in memory CD8 + T cells after TAK-779 treatment in NP-immunized mice, it is useful to compare their functional characteristics upon antigen encounter. We evaluated their ability to produce effector cytokines upon in vitro restimulation. We found that NP-immunized mice treated with TAK-779 exhibited significantly higher numbers of IFN- $\gamma-$ producing cells than their non-TAK-779 treated counterparts, and this difference became even greater when mice were immunized twice with NP (Figures 7A and $\mathrm{B})$. Our data demonstrates that CD8 + T cells generated after TAK-779 treatment in NP-immunized mice are fully functional and able to exert their effector functions upon antigen restimulation.

TAK-779-Treated NP-Immunized Mice Showed Enhanced Protection after Infection with Heterologous Influenza A Virus

To examine whether the increased number of memory CD8 $+\mathrm{T}$ cells in TAK-779-treated NP-immunized mice correlated with enhanced protection compared with NP-immunized mice without TAK-779 treatment, we challenged the different groups of mice with heterologous influenza A virus (H1N1 (A/FM/1/47)) at 10LD50. As shown in Figures $8 \mathrm{~A}$ and $\mathrm{B}$, although all groups of mice succumbed to the high-dose heterologous influenza virus challenge, a significant delay in death and changes in body weight were observed in mice immunized twice with NP and treated with TAK-779 (TN2 group) compared with those in the control and NP groups (naïve, TAK, NP and NP2) $(P \leq 0.05)$

(Figures 8A and B). However, there was 
A

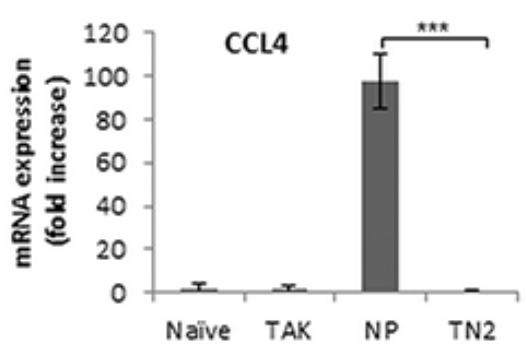

C

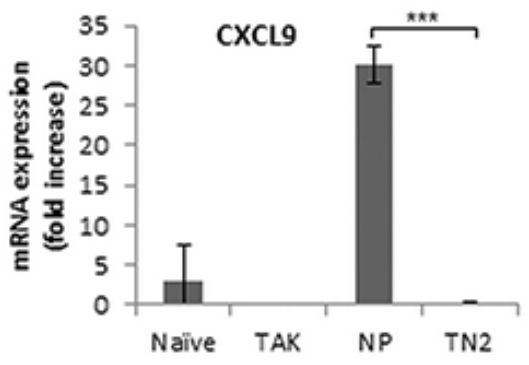

E

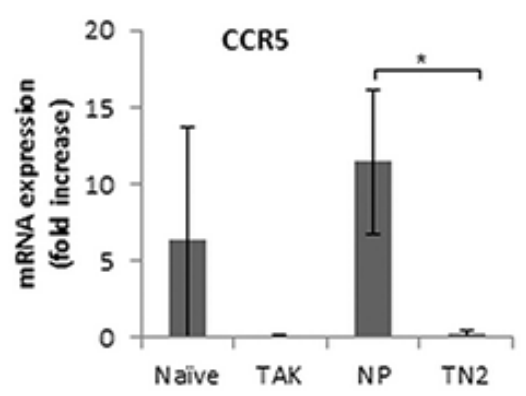

B

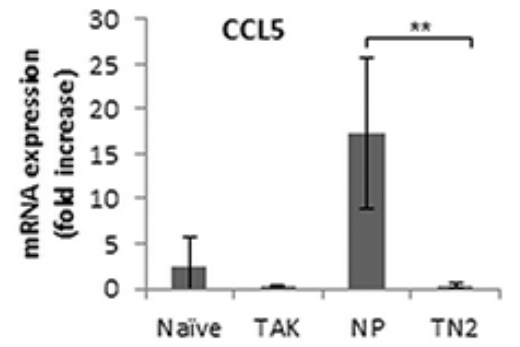

D

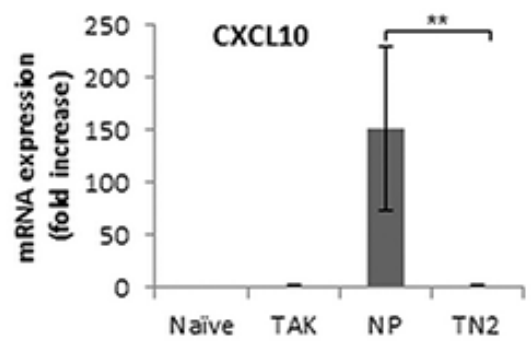

F

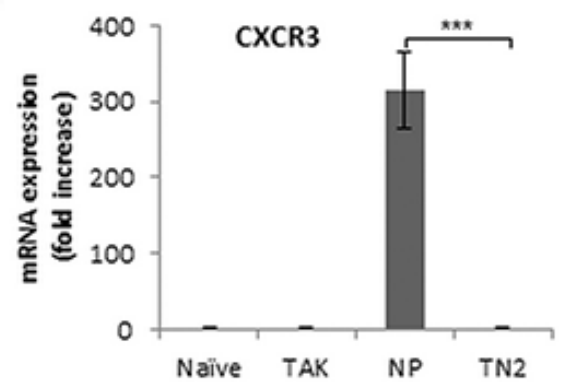

the recall response (Figure 9). Taken together, our data demonstrate that TAK779 administration in mice immunized twice with NP displayed increased memory generation and enhanced protection after infection with heterologous influenza A virus, leading to prolonged survival and decreased viral loads during the early stages of infection without deterioration of lung immunopathology.

\section{DISCUSSION}

Since it is generally agreed that success against major human pathogens such as HIV, Mycobacterium tuberculosis and Plasmodium falciparum will likely require induction of very large memory CD8 + T cell responses, some of the most promising human vaccines under development are targeted at augmenting both the number and function of memory CD8 + T cells. (28) However, the ability to generate protective memory $\mathrm{CD} 8+\mathrm{T}$ cells has proven more complicated and problematic. This study assessed the effect on host immune response of temporarily dampening the chemokine receptors CXCR3 and CCR 5 after vaccination by administration of TAK-779. Our results show that use of the small-molecule antagonist TAK-779 to block chemokine receptors CXCR3 and CCR5 can indeed enhance memory CD8 + T cell immune response both qualitatively and quantitatively. These memory $\mathrm{T}$ cells were able to become IFN- $\gamma$-secreting effector cells when re-encountering the same antigen, which can further enhance the protection efficacy of vaccination during infection.

A number of studies have revealed the profound relationship between low-inflammation scenarios of CD8 + $\mathrm{T}$ cell priming and the magnitude and quality of CD8 + T cell effector and memory response. (5,29-31) Chemokine receptors have been shown to play an important role in many inflammatory situations, primarily by directing the migration of immune cells. Recent studies have demonstrated that antigen-specific $\mathrm{CD} 8+\mathrm{T}$ cells in the lungs that lacked the inflammatory chemokine receptor CXCR3 showed decreased activation, 
A

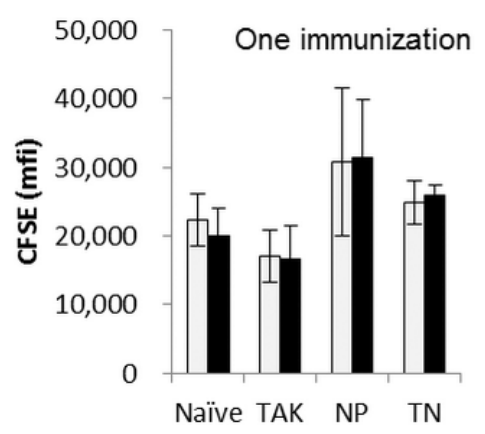

B

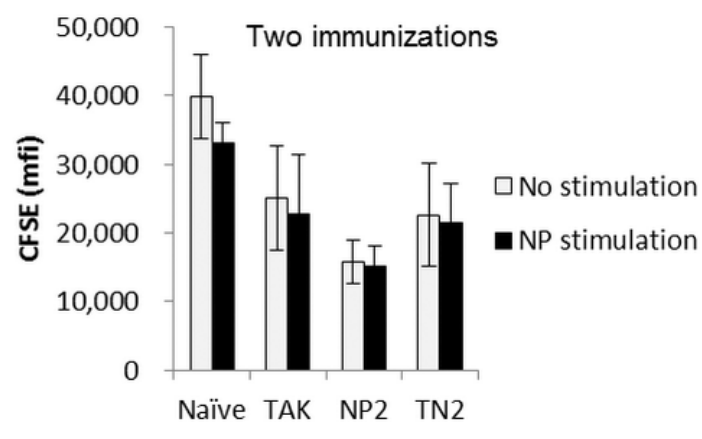

Figure 6. Proliferative ability of CD8 + T cells in NP-immunized mice with or without TAK-779 treatment compared with control group. Two weeks after TAK-779 cessation, splenocytes were prepared from individual spleens collected from naïve mice, mice treated with TAK-779 (TAK), mice immunized once (NP) or twice with NP (NP2) and mice immunized once (TN) or twice (TN2) with NP and treated with TAK-779. Proliferation was measured as mean fluorescence intensity ( $\mathrm{mfi}$ ) of CFSE by flow cytometry upon NP stimulation. (A) One NP immunization; (B) 2 NP immunizations. Results are expressed as the mean \pm SD. The data are representative of 2 independent experiments with at least 4 mice per group. Mice were analyzed individually.

A

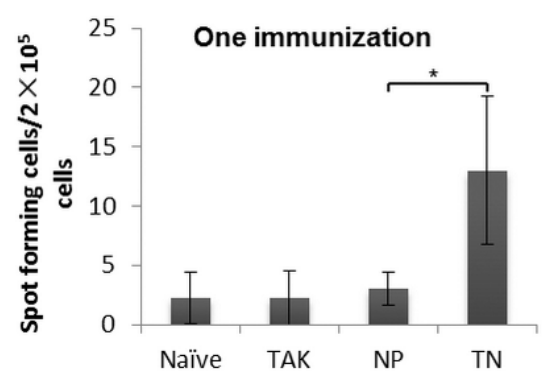

B

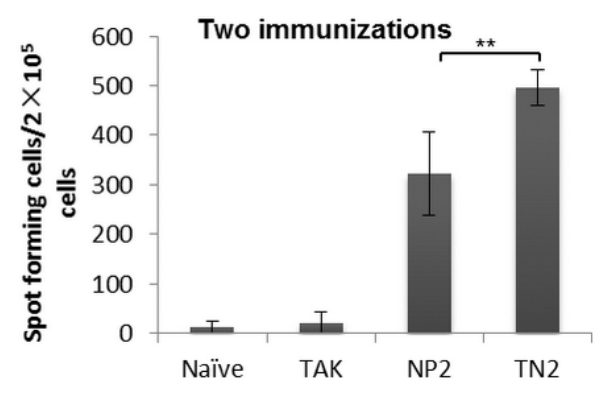

Figure 7. Effector functional characteristics of cells in NP-immunized mice with or without TAK-779 treatment compared with control group. Two weeks after TAK-779 cessation, individual spleens were collected from naïve mice, mice treated with TAK-779 (TAK), mice immunized once (NP) or twice with NP (NP2) and mice immunized once (TN) or twice (TN2) with NP and treated with TAK-779. Splenocytes were prepared and analyzed by IFN- $\gamma$ ELISPOT assay upon restimulation with NP protein. Results are expressed as the mean \pm SD of the number of positive spots per $2 \times 10^{5}$ cells. Results are representative of 2 independent experiments. ${ }^{*} p \leq 0.05,{ }^{* *} p \leq 0.001$.

were protected from apoptosis and were largely resistant to contraction, leading to the generation of massive amounts of CD8 + T cell memory. (11-13) CCR5 was also shown to play a role in the enhancement of T cell memory. (13) On the other hand, since several chemokine receptors, including CXCR3 and CCR5, have been shown to play a role in the accumulation of effector $\mathrm{T}$ cells in the lung, $(32,33)$ permanent inhibition of
CXCR3 and CCR5 may be detrimental to the host. Using the CXCR3 and CCR5 antagonist TAK-779, we showed that NP vaccination along with temporary TAK-779 treatment resulted in enhanced memory generation, with more memory precursor and fewer terminally differentiated effector CD8 + T cells. Significantly more CD127 + and fewer CD69 + CD8 T cells were detected in NP-immunized TAK-779-treated mice than their non-TAK-779-treated counterparts as well (Figure 4). In addition, as many of the signals that shape CD8 + T cell memory have the potential to affect other aspects of the adaptive (B cells and CD4 + T cells) or innate immune response, we also examined humoral immune response after TAK-779 administration. However, no significant differences in total anti-NP IgG and its isotypes were found between the immunized groups with or without TAK-779 treatment. Taken together, our data demonstrate that TAK-779 treatment after vaccination significantly expedited cell-mediated response to an immunized antigen with no negative effect on humoral recall response. Interestingly, being the first licensed antiretroviral therapeutic agent that targets CCR5, Maraviroc (MVC) has also been shown to have immunomodulatory effects on vaccination response, T-cell phenotype and function in HIV-1+ subjects. (34) MVC intensification has a favorable immunological impact on $\mathrm{T}$ cell phenotype and cell-mediated responses to previously encountered antigens, leading to increased memory $\mathrm{T}$ cells and decreased CD8 + T cell activation. (34) Kavana-Tachikawa et al. also demonstrated that MVC beneficially impacts host HIV-1-specific T cell immunity with prolonged maintenance of virus-specific T cell response. (35) Hence, our study provides further data on the immunomodulatory and beneficial use of MVC containing cART in HIV infection, and potentially in other immunologically relevant settings.

Although TAK-779 treatment resulted in quantitative and phenotypic differences in CD8 + T cells, it did not enhance the proliferative ability of CD8 $+\mathrm{T}$ cells (Figure 5). Thus the significant increase in number of memory CD8 $+\mathrm{T}$ cells could be a consequence of attenuated contraction. (13) We further show that CD8 + T cells generated after TAK-779 treatment in NP-immunized mice are fully functional and able to exert their effector functions upon antigen restimulation in vitro (Figure 6). Indeed, viral loads were 
A

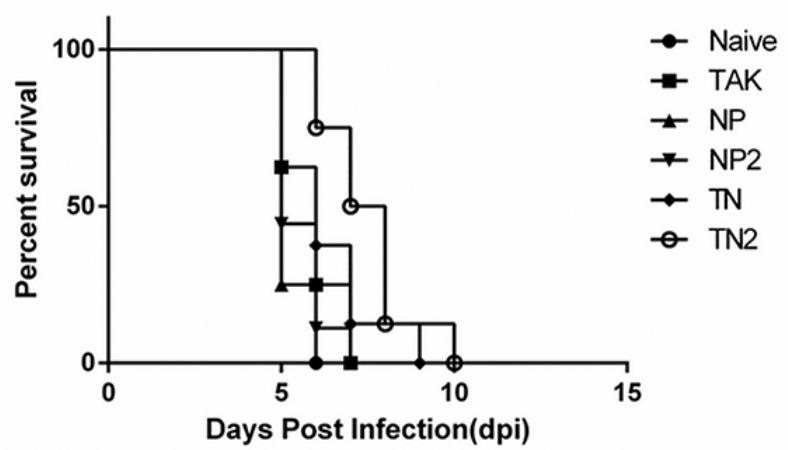

B

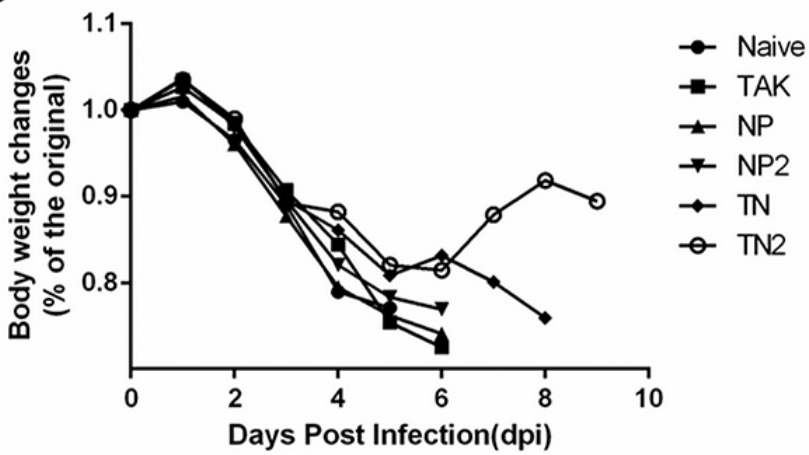

C

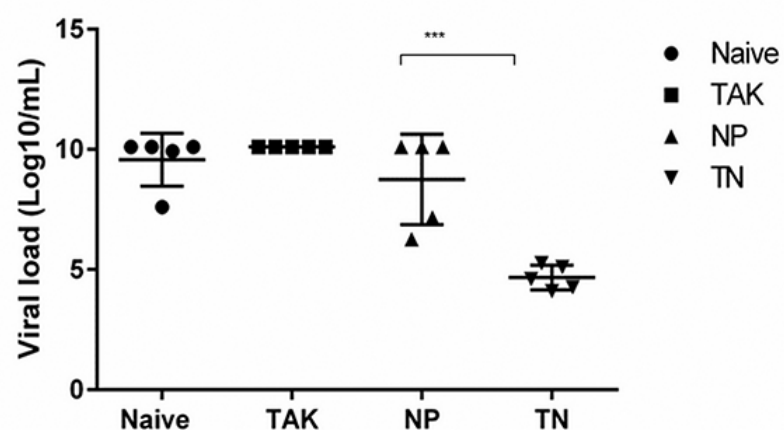

Figure 8. Protection efficacies of mice immunized with NP with or without TAK-779 treatment against lethal H1N1 influenza A virus challenge Mice were immunized once or twice with NP and treated with or without TAK-779 as scheduled. Two weeks after TAK-779 cessation, mice were challenged with 10 LD50 of HIN1 (A/FM/1/47). The survival rates (A) were compared with infected control mice (naivve and TAK groups). Body-weight changes (B) were monitored daily, and mice were euthanized when body weight loss exceeded $25 \%$ of original body weight. (C) Viral load quantification in the lungs of control mice and mice immunized twice with NP and treated with or without TAK-779. Mice were immunized twice with NP and treated with or without TAK-779 as scheduled. Two weeks after TAK-779 cessation, mice were challenged with 10 LD50 of HINI (A/FM/1/47). At 3 d post-viral challenge, the viral loads were measured in the lungs of different groups of mice by TCID50 assay. Each symbol represents an individual mouse, and the lines represent the geometric mean \pm SD. Five animals per group per time point were assayed. ${ }^{* *} p \leq 0.001$.

significantly reduced in NP2 + TAK mice vs control mice and NP2 mice at $\mathrm{d} 3$ post influenza virus challenge without deteriorating lung immunopathology
(Figures 7C and 8). However, when the mice were challenged with 10LD50 heterologous H1N1 (A/FM/1/47) influenza virus, protection was suboptimal and all groups of mice succumbed to infection. This could be attributed to the protective efficacy of memory CD8 + cells against influenza A virus infection. Actually, the contribution of memory CD8 $+\mathrm{T}$ cells to protection against influenza $\mathrm{A}$ virus is still under debate, because several studies have shown that vaccination or previous influenza A virus exposure did not protect from subsequent heterologous influenza A virus infection. (36-38) Another reason may be that antigen-specific memory CD8 $+\mathrm{T}$ cells generated in the study were still not enough to confer optimal protection, or the virus dose used for the challenge was too high, and further improvement of the study is a matter of future research. Nevertheless, mice immunized twice with NP and treated with TAK-779 (NP2 + TAK group) indeed showed significant delay in death and changes in body weight compared with those in the control groups and NP groups (naïve, TAK, NP and NP2) $(\mathrm{P}<0.05)$, demonstrating that the significant increase in memory CD8 $+\mathrm{T}$ cells by temporary CXCR3 and CCR5 inhibition with TAK-779 administration was translated into better protection upon influenza A virus challenge.

As mentioned earlier, chemokines and their receptors have been reported as essential and selective mediators in leukocyte migration to inflammatory sites and secondary lymphoid organs. As a G-protein-coupled chemotactic receptor, CXCR3 has been found to affect tissue recruitment of antiviral CD8 + T cells and antiviral immunity. $(39,40)$ Studies have shown that during secondary viral infection, CXCR3 expression by memory effector CD8 + T cells in the lymph nodes is needed for $\mathrm{T}$ cell trafficking to infected peripheral tissues. $(41,42)$ Recently, CXCR3 has been shown to be critical for memory CD8 + T cells to populate the airways and for virus-infected cells to exert antiviral effector functions during a steady state of influenza A virus infection. $(43,44)$ In addition, Kohlmeier and colleagues discovered that CCR5 played a crucial role in the accelerated recruitment of memory $\mathrm{CD} 8+\mathrm{T}$ cells to the lung airways during 


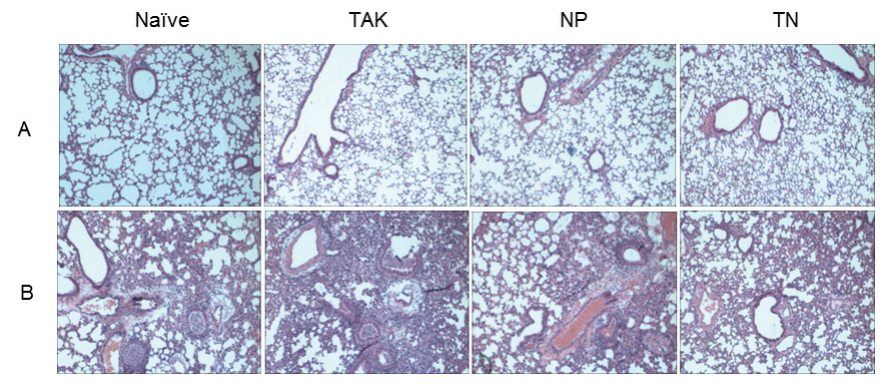

Figure 9. Lung histopathology of the lungs of control mice and mice immunized twice with NP and treated with or without TAK-779. Mice were immunized twice with NP and treated with or without TAK-779 as scheduled. Two weeks after TAK-779 cessation, mice were challenged with 10 LD50 of HIN1 (A/FM/1/47). At 3 d post-viral challenge, all infected mice displayed significant perivascular and peribronchial inflammation, pulmonary edema and necrotizing bronchitis. Forty fields were analyzed per group ( $>5$ fields/ section, 2 sections/mouse). Representative sections are shown. The data were representative of 2 independent experiments with 5 mice per group.

influenza virus challenge, and CCR5 deficiency resulted in decreased recruitment of memory $\mathrm{T}$ cells and impaired control of virus replication during the initial stages of a secondary response. (45) In our study, since TAK-779 treatment was ceased 2 wks before the influenza virus challenge, CXCR3 and CCR5 were only temporarily dampened, and this would not have affected subsequent CD8 + T cell migration to the airways to carry out their effector functions. Further studies are necessary to determine if restoration of CXCR3 and CCR5 expression on CD8 $+\mathrm{T}$ cells occurs after an influenza virus challenge. Furthermore, the redundancy in expression of inflammatory chemokine receptors such as CXCR6 may facilitate the recruitment, activation and function of memory CD8 $+\mathrm{T}$ cells in a secondary response.

Some limitations of the study merit discussion. Due to the availability of TAK-779 and the necessity of using relevant control groups, we were not able to optimize the minimum duration of TAK-779 treatment that is required for protection. Challenge with a lower dose of influenza virus was also hampered by TAK-779 availability, and this hindered us in observing the protective efficacy more clearly. In addition, it would be interesting to test the protective efficacy of temporary CXCR3 and CCR5 inhibition during other vaccination scenarios in future studies.
In conclusion, our study demonstrates that temporary inhibition of chemokine receptors CXCR3 and CCR5 through TAK-779 administration leads to enhanced memory CD8 + T cell immune response and better protection against a lethal influenza A virus challenge. Our study is of particular importance to vaccination against pathogens against which high numbers of memory CD8 + T cells are needed. Our study also supports the immunomodulatory and beneficial use of MVC containing cART in HIV infection and potentially in other immunologically relevant settings.

\section{CONCLUSION}

Temporarily dampening the chemokine receptors CXCR3 and CCR5 during vaccination by administration of TAK779 resulted in enhanced memory CD8 + T cell immune response, with more memory precursor and fewer terminally differentiated effector cells. These memory $\mathrm{T}$ cells were able to become IFN- $\gamma$-secreting effector cells upon antigen restimulation, which can further enhance the efficacy of vaccination. These data provide a promising strategy for vaccination against pathogens where high numbers of memory CD8 + T cells are needed, and also supports the immunomodulatory and beneficial use of MVC containing cART.

\section{ACKNOWLEDGEMENTS}

We gratefully thank Dr. Zhisong Chen for providing TAK-779 (NIH) and Prof. Sylvie Alonso (Department of Microbiology, National University of Singapore) for her critical reading of and useful comments on the manuscript.

\section{DISCLOSURE}

This work was supported by the National Natural Science Foundation of China (individual research grants allocated to Dr Rui Li, KRH1322418), the Department of Microbiology and Microbial Engineering, Fudan University (startup grant allocated to Dr Li, JJH1322015).

\section{REFERENCES}

1. Schmidt NW, et al. (2008) Memory CD8 T cell responses exceeding a large but definable threshold provide long-term immunity to malaria. Proc. Natl. Acad. Sci. U.S.A. 105:14017-22.

2. Williams MA, Bevan MJ. (2007) Effector and memory CTL differentiation. Annu. Rev. Immunol. 25:171-92.

3. Badovinac VP, Harty JT. (2006) Programming, demarcating, and manipulating CD8+ T-cell memory. Immunol. Rev. 211:67-80.

4. Harty JT, Badovinac VP. (2008) Shaping and reshaping CD8+ T-cell memory. Nat. Rev. Immunol. 8:107-19.

5. Butler NS, Nolz JC, Harty JT. (2011) Immunologic considerations for generating memory CD8 T cells through vaccination. Cell Microbiol. 13:925-33.

6. Joshi NS, et al. (2007) Inflammation directs memory precursor and short-lived effector CD8(+) T cell fates via the graded expression of T-bet transcription factor. Immunity. 27:281-95.

7. Kalia V, et al. (2010) Prolonged interleukin2Ralpha expression on virus-specific CD8+ T cells favors terminal-effector differentiation in vivo. Immunity. 32:91-103.

8. Pipkin ME, et al. (2010) Interleukin-2 and inflammation induce distinct transcriptional programs that promote the differentiation of effector cytolytic T cells. Immunity. 32:79-90.

9. Rubinstein MP, et al. (2008) IL-7 and IL-15 differentially regulate CD8+ T-cell subsets during contraction of the immune response. Blood. 112:3704-12.

10. Sandau MM, Kohlmeier JE, Woodland DL, Jameson SC. (2010) IL-15 regulates both quantitative and qualitative features of the memory CD8 T cell pool. J. Immunol. 184:35-44.

11. Kurachi M, et al. (2011) Chemokine receptor CXCR3 facilitates CD8(+) T cell differentiation into short-lived effector cells leading to memory degeneration. J. Exp. Med. 208:1605-20.

12. Hu JK, Kagari T, Clingan JM, Matloubian M. (2011) Expression of chemokine receptor CXCR3 
on $\mathrm{T}$ cells affects the balance between effector and memory CD8 T-cell generation. Proc. Natl. Acad. Sci. U.S.A. 108: E118- E127.

13. Kohlmeier JE, et al. (2011) Inflammatory chemokine receptors regulate CD8(+) T cell contraction and memory generation following infection. J. Exp. Med. 208:1621-34.

14. Baba M, et al. (1999) A small-molecule, nonpeptide CCR5 antagonist with highly potent and selective anti-HIV-1 activity. Proc. Natl. Acad. Sci. U.S.A. 96:5698-5703.

15. Gao P, et al. (2003) The unique target specificity of a nonpeptide chemokine receptor antagonist: selective blockade of two Th1 chemokine receptors CCR5 and CXCR3. J. Leukoc. Biol. 73:273-80.

16. Takama $Y$, et al. (2011) Effects of a calcineurin inhibitor, FK506, and a CCR5/CXCR3 antagonist, TAK-779, in a rat small intestinal transplantation model. Transpl. Immunol. 25:49-55.

17. Bastani S, et al. (2009) Chemokine Receptor Blockade With a Synthetic Nonpeptide Compound Attenuates Cardiac Allograft Vasculopathy. Transplantation. 88:995-1001.

18. Kakuta Y, et al. (2012) Blocking of CCR5 and CXCR3 Suppresses the Infiltration of Macrophages in Acute Renal Allograft Rejection. Transplantation. 93:24-31.

19. Tokuyama H, et al. (2005) The simultaneous blockade of chemokine receptors CCR2, CCR5 and CXCR3 by a non-peptide chemokine receptor antagonist protects mice from dextran sodium sulfate-mediated colitis. Int. Immunol. 17:1023-34.

20. Suzaki Y, et al. (2008) A small-molecule compound targeting CCR5 and CXCR3 prevents airway hyperresponsiveness and inflammation. Eur. Respir. J. 31:783-89.

21. Ni J, et al. (2009) The chemokine receptor antagonist, TAK-779, decreased experimental autoimmune encephalomyelitis by reducing inflammatory cell migration into the central nervous system, without affecting $\mathrm{T}$ cell function. Br. J. Pharmacol. 158:2046-56.

22. Yang YF, et al. (2002) A non-peptide CCR5 antagonist inhibits collagen-induced arthritis by modulating $\mathrm{T}$ cell migration without affecting anti-collagen $\mathrm{T}$ cell responses. Eur. J. Immunol. 32:2124-32.

23. Akashi S, et al. (2005) A novel small-molecule compound targeting CCR5 and CXCR3 prevents acute and chronic allograft rejection. Transplantation. 80:378-84.

24. Quah BJ, Warren HS, Parish CR. (2007) Monitoring lymphocyte proliferation in vitro and in vivo with the intracellular fluorescent dye carboxyfluorescein diacetate succinimidyl ester. Nat. Protoc. 2:2049-56.

25. Li R, et al. (2010) Attenuated Bordetella pertussis protects against highly pathogenic influenza A viruses by dampening the cytokine storm. J. Virol. 84:7105-13.

26. Kaech SM, Wherry EJ. (2007) Heterogeneity and cell-fate decisions in effector and memory CD8+
T cell differentiation during viral infection. Immunity. 27:393-405.

27. Rutishauser RL, Kaech SM. (2010) Generating diversity: transcriptional regulation of effector and memory CD8 T-cell differentiation. Immunol. Rev. 235:219-33.

28. Good MF, Doolan DL. (2010) Malaria vaccine design: immunological considerations. Immunity. 33:555-66.

29. Badovinac VP, Porter BB, Harty JT. (2002) Programmed contraction of CD8(+) T cells after infection. Nat. Immunol. 3:619-26.

30. Zehn D, Lee SY, Bevan MJ. (2009) Complete but curtailed T-cell response to very low-affinity antigen. Nature. 458:211-14.

31. Kaech SM, Ahmed R. (2001) Memory CD8+ T cell differentiation: initial antigen encounter triggers a developmental program in naive cells. Nat. Immunol. 2:415-22.

32. Kohlmeier JE, et al. (2009) CXCR3 directs antigen-specific effector CD4+ T cell migration to the lung during parainfluenza virus infection. J. Immunol. 183:4378-84.

33. Galkina E, et al. (2005) Preferential migration of effector CD8+ T cells into the interstitium of the normal lung. J. Clin. Invest. 115:3473-83.

34. Westrop SJ, et al. (2012) CCR5 antagonism impacts vaccination response and immune profile in HIV-1 infection. Mol. Med. 18:1240-48.

35. Kawana-Tachikawa A, et al. (2014) Effect of maraviroc intensification on HIV-1-specific T cell immunity in recently HIV-1-infected individuals. PLoS One. 9: e87334.

36. Steinhoff MC, Fries LF, Karron RA, Clements ML, Murphy BR. (1993) Effect of heterosubtypic immunity on infection with attenuated influenza A virus vaccines in young children. J. Clin. Microbiol. 31:836-38.

37. McMichael AJ, Gotch FM, Noble GR, Beare PA. (1983) Cytotoxic T-cell immunity to influenza. N. Engl. J. Med. 309:13-17.

38. Wilkinson TM, et al. (2012) Preexisting influenza-specific CD4+ T cells correlate with disease protection against influenza challenge in humans. Nat. Med. 18:274-80.

39. Hokeness KL, et al. (2007) CXCR3-dependent recruitment of antigen-specific $\mathrm{T}$ lymphocytes to the liver during murine cytomegalovirus infection. J. Virol. 81:1241-50.

40. Lee BJ, et al. (2005) Role of CXCR3 in the immune response to murine gammaherpesvirus 68 . J. Virol. 79:9351-55.

41. Kastenmuller W, et al. (2013) Peripheral prepositioning and local CXCL9 chemokine-mediated guidance orchestrate rapid memory CD8+ T cell responses in the lymph node. Immunity. 38:502-13.

42. Sung JH, et al. (2012) Chemokine guidance of central memory $\mathrm{T}$ cells is critical for antiviral recall responses in lymph nodes. Cell. 150:1249-63.

43. Hickman HD, et al. (2015) CXCR3 chemokine receptor enables local CD8(+) T cell migration for the destruction of virus-infected cells. Immunity. 42:524-37.

44. Slutter B, Pewe LL, Kaech SM, Harty JT. (2013) Lung airway-surveilling CXCR3(hi) memory CD8(+) T cells are critical for protection against influenza A virus. Immunity. 39:939-48.

45. Kohlmeier JE, et al. (2008) The chemokine receptor CCR5 plays a key role in the early memory CD8+ T cell response to respiratory virus infections. Immunity. 29:101-13.

Cite this article as: Li R, et al. (2016) Temporary CXCR3 and CCR5 antagonism following vaccination enhances memory CD8 $\mathrm{T}$ cell immune responses. Mol. Med. 22:497-507. 